\title{
Filter Banks from the Fibonacci Sequence
}

\author{
Fuxian Chen $^{1}$, Qiuhui Chen ${ }^{1}$, Weibin Wu ${ }^{2}$, Xiaoming Wang ${ }^{2}$ \\ ${ }^{1}$ Faculty of Mathematics and Informatics, South China Agricultural University, Guangzhou, China \\ ${ }^{2}$ Faculty of Engineer, South China Agricultural University, Guangzhou, China
}

\section{Email address:}

scaufuxian@outlook.com (Fuxian Chen), chenqiuhui@hotmail.com (Qiuhui Chen), wuweibin@ scau.edu.cn (Weibin Wu), $58339843 @$ qq.com (Xiaoming Wang)

\section{To cite this article:}

Fuxian Chen, Qiuhui Chen, Weibin Wu, Xiaoming Wang. Filter Banks from the Fibonacci Sequence. Pure and Applied Mathematics Journal. Vol. 8, No. 6, 2019, pp. 100-105. doi: 10.11648/j.pamj.20190806.12

Received: October 21, 2019; Accepted: December 2, 2019; Published:Published: December 31, 2019

\begin{abstract}
Wavelet transform is an important quadratic representation in time-frequency domain of signals. The main advantage of wavelet transform is the time frequency localization as compared with the fourier transform. Due to the reason of dilation and translation operation acting the basic time-frequency atoms. Therefore a multi-resoloution analysis strategy is devoted to the construction of wavelet basis of $L^{2}(R)$, which also establishes a bridge between engineer and mathematics. The construction of wavelets is equivalent to the design of filter banks with complete reconstruction. In this note we investigate filter banks from the Fibonacci sequence. The draw back is that, the convergence $z$-transform is less than 1, hence it can not be used as filter.By adopting the Hadamard product of the Fibonacci sequence and a geometric sequence, a type of Fibonacci-based bi-orthogonal filter banks are constructed. This kind of filter banks are based two bricks: Bezout polynomials and the mask of the cardinal B-splines. These filters are essentially rational functions, which have potential applications in system identification and signal processing.
\end{abstract}

Keywords: Filter Banks, Bezout Polynomial, Wavelet, Symbol of B-spline

\section{Introduction}

The Fibonacci sequence is defined by the rule that every number after the first two is the sum of the two preceding ones:

$$
1,1,2,3,5,8,13,21,34,55,89,144,233 \ldots,
$$

which holds the recurrence relation

$$
F_{n+2}=F_{n}+F_{n+1}
$$

with initial values $F_{0}=F_{1}=1$.

The Fibonacci sequence appears often unexpectedly in mathematics so that there is an entire journal dedicated to its study, the Fibonacci Quarterly. Applications of the Fibonacci sequence includes computer algorithms such as search technique and data systems [11-13]. It also appears in biological setting[14, 15].

In this note, we will establish a relationship between the Fibonacci sequence and filter banks. Multiplication by $z^{n}$ and summation over $n$ on the both sides of (1), it gives rise to the $z$-transform of the Fibonacci sequence

$$
F(z)=\sum_{n=0}^{\infty} F_{n} z^{n}=\frac{1}{1-z-z^{2}}, z \in \mathbb{C} .
$$

Note that the convergence radius of above series is $\frac{\sqrt{5}-1}{2}$ and then the series $\sum_{k} F_{k}$ is not convergent. This fact indicates that the Fibonacci sequence is not a low-pass filter. Here a sequence $\left\{c_{k}: k \in \mathbb{Z}\right\}$ is called a low-pass filter if its $z$ transform $c(z)$ satisfies the requirement $c(1)=1$. In order to design a kind of low-pass filter from the Fibonacci sequence, we consider the Hadamard product of the Fibonacci sequence and the geometric sequence $\left(\lambda^{n}: n=0,1,2, \ldots\right)$, that is, a new sequence

$$
\left(1, \lambda, 2 \lambda^{2}, 3 \lambda^{3}, 5 \lambda^{4}, 8 \lambda^{5}, 13 \lambda^{6}, 21 \lambda^{7}, 34 \lambda^{8}, \cdots\right) .
$$


A normalization offers the following sequence

$\left(1-\lambda-\lambda^{2}\right)\left(1, \lambda, 2 \lambda^{2}, 3 \lambda^{3}, 5 \lambda^{4}, 8 \lambda^{5}, 13 \lambda^{6}, 21 \lambda^{7}, 34 \lambda^{8}, \cdots\right)$.

$$
a_{n}=\left(1-\lambda-\lambda^{2}\right) \lambda^{n} F_{n}=\frac{\lambda^{2}+\lambda-1}{\sqrt{5}}\left(\left(\frac{1-\sqrt{5}}{2}\right)^{n+1} \lambda^{n}-\left(\frac{1+\sqrt{5}}{2}\right)^{n+1} \lambda^{n}\right)
$$

It satisfies the recursive formula $a_{n+2}=\lambda^{2} a_{n}+\lambda a_{n+1}$ and holds $\sum_{n} a_{n}=1$. The $z$-transform of $\left\{a_{n}\right\}$ is the rational function

$$
h_{0}(z)=\frac{\lambda^{2}+\lambda-1}{\lambda^{2} z^{2}+\lambda z-1}, z \in\left\{z \in \mathbb{C}:|z|<\frac{\sqrt{5}-1}{2 \lambda}\right\} .
$$

With the restriction $0<\lambda<\frac{\sqrt{5}-1}{2}$, it gives $h_{0}(1)=1$.

For any fixed positive integer $N$, we will investigate the lowpass filter

$$
\begin{aligned}
& H_{0}(z)=\left(\frac{1+z}{2}\right)^{N} h_{0}(z) . \\
& m_{0}(\xi)=\left.H_{0}(z)\right|_{z=e^{-i \xi} \ell_{N}}(\xi)=\left(\frac{1+e^{-i \xi}}{2}\right)^{N} \frac{\lambda^{2}+\lambda-1}{\lambda^{2} e^{-2 i \xi}+\lambda e^{-i \xi}-1} \ell_{N}(\xi), \xi \in \mathbb{R},
\end{aligned}
$$

Which holds $m_{0}(0)=1$. The low-pass property of $H_{0}$ or $m_{0}$ (i.e. $H_{0}(1)=1$ or $m_{0}(0)=1$ ) suggests us to investigate the intrinsic structure of the corresponding filter banks with perfect reconstruction and the wavelet functions.

The first filter banks with two channels were introduced by Croisier, Esteban, and Galand [2] in 1976 when they compressed speech signal by subband coding schemes, which decomposes a discrete signal into two signals of half its size by using a filtering and subsampling procedure. These research showed that the signal can be recovered from these subsampled signals by canceling the aliasing terms with a particular class of filters called conjugate mirror filters $(\mathrm{CMF})$. This breakthrough motivated an active

$$
\left(\begin{array}{cc}
m_{0}(\xi) & m_{0}(\xi+\pi) \\
m_{1}(\xi) & m_{1}(\xi+\pi)
\end{array}\right)\left(\begin{array}{c}
\tilde{m}_{0}(\xi) \\
\tilde{m}_{1}(\xi)
\end{array}\right.
$$

$\tilde{m}_{1}$

In this case, we also say that $\left(m_{0}, m_{1}, \tilde{m}_{0}, \tilde{m}_{1}\right)$ forms perfect reconstruction filter banks. The functions $m_{0}, m_{1}$ are called decomposition filters and correspondingly the dual $\tilde{m}_{0}, \tilde{m}_{1}$ are addressed synthesis filters. In subband filtering,
We remark that the factor $\left(\frac{1+z}{2}\right)^{N}$ controls the regularity of scaling functions, which will be explained in the next section.

An equivalent form of the filter $H_{0}$ is its boundary value on research effort to build a complete filter bank theory. Necessary and sufficient conditions for decomposing a signal in subsampled components with a filtering scheme, and recovering the same signal with an inverse transform, were established by Smith and Barnwell [9]. Daubechies [3] designed univariate two-channel perfect reconstruction filter banks having finite impulse response (FIR) corresponding to univariate orthonormal wavelet having compact support and vanishing moments. According to the theory of subband filtering and multiresolution analysis[7], in the two channel case, a filter bank is the vector $\left(m_{0}, m_{1}, \tilde{m}_{0}, \tilde{m}_{1}\right)$ with entries being $2 \pi$-periodic functions, which satisfies the matrix equation

$$
\left.\begin{array}{c}
\tilde{m}_{0}(\xi+\pi) \\
\tilde{m}_{1}(\xi+\pi)
\end{array}\right)^{*}=I_{2 \times 2}, \text { a.e. } \xi \in[-\pi, \pi] .
$$

one emphasizes the frequency decomposition of signals, that is, $m_{0}$ is a low-pass filter with $m_{0}(0)=1$ and $m_{1}$ is a highpass filter with $m_{1}(\pi)=0$.

The equation (5) is equivalent to find $\tilde{H}_{0}, H_{1}, \tilde{H}_{1}$ such that

$$
\left(\begin{array}{cc}
H_{0}(z) & H_{0}(-z) \\
H_{1}(z) & H_{1}(-z)
\end{array}\right)\left(\begin{array}{cc}
\tilde{H}_{0}(z) & \tilde{H}_{0}(-z) \\
\tilde{H}_{1}(z) & \tilde{H}_{1}(-z)
\end{array}\right)^{*}=I_{2 \times 2}, z \in \partial \mathbb{D} .
$$

Here, $\mathbb{D}$ is the unit disc $\{z:|z|<1\}$ and $\partial \mathbb{D}$ is its boundary.

Under suitable conditions on $m_{0}$, one can define refinable functions and wavelets in $L^{2}(\mathbb{R})$ through the inverse Fourier transform by the approach of infinite product

$$
\hat{\phi}(\xi):=\frac{1}{\sqrt{2 \pi}} \prod_{k=1}^{\infty} m_{0}\left(\xi / 2^{k}\right), \quad \hat{\tilde{\phi}}(\xi):=\frac{1}{\sqrt{2 \pi}} \prod_{k=1}^{\infty} \tilde{m}_{0}\left(\xi / 2^{k}\right), \text { a.e. } \xi \in \mathbb{R},
$$

and

$$
\hat{\psi}(\xi):=m_{1}(\xi / 2) \hat{\phi}(\xi / 2), \quad \hat{\tilde{\psi}}(\xi):=\tilde{m}_{1}(\xi / 2) \hat{\tilde{\phi}}(\xi / 2), \text { a.e. } \xi \in \mathbb{R} \text {. }
$$


Throughout this paper, the Fourier transform $\hat{g}=\mathcal{F} g$ of $g \in L^{1}(\mathbb{R})$ is defined at $\xi \in \mathbb{R}$ by the equation

$$
(\mathcal{F} g)(\xi):=\frac{1}{\sqrt{2 \pi}} \int_{\mathbb{R}} g(t) e^{-i \xi t} d t
$$

The Plancherel theorem allows us to extend the definition of the Fourier transform to any function in $L^{2}(\mathbb{R})$ by the density arguments. The Fourier transform has an extension for even tempered distribution.

We call $\phi$ and $\tilde{\phi}$ scaling functions, and $\psi$ and $\tilde{\psi}$ wavelets, respectively. Note that the scaling function $\phi$ defined by the infinite product $\prod_{k=1}^{\infty} m_{0}\left(\xi / 2^{k}\right)$ holds the identity

$$
\hat{\phi}(\xi)=m_{0}(\xi / 2) \hat{\phi}(\xi / 2), \text { a.e. } \xi \in \mathbb{R} .
$$

A inverse Fourier transform gives rise to the refinement equation

$$
\phi(t)=\sum_{k \in \mathbb{Z}} a_{k} \phi(2 t-k), t \in \mathbb{R}
$$

where $\left\{a_{k}: k=0,1,2, \cdots\right\}$ is the sequence of the coefficients of the low-pass filter $m_{0}$ defined in (4).

We need to revisit Bezout polynomials and cardinal Bsplines because they are crucial for the design of our IIR (infinite impulse response) filter banks with perfect reconstruction.

\section{Review of Bezout Polynomials and B-splines}

The Bezout polynomial and the symbol of cardinal Bspline are basic bricks for the design of our filter banks. The Bezout polynomial plays a crucial role in the construction of Daubechies wavelets, which is defined by

$$
P_{N}(x)=\sum_{k=0}^{N-1}\left(\begin{array}{c}
N+k-1 \\
k
\end{array}\right) x^{k}, x \in \mathbb{R} .
$$

The Bezout polynomial is the unique lowest degree solution of the equation

$$
(1-x)^{N} P(x)+x^{N} P(1-x)=1, x \in \mathbb{R} .
$$

Essentially, $P_{N}$ is the first @ terms truncation of the Taylor expansion of $(1-x)^{-N}$.

We need a lemma by Riesz, which indicates that one can extract the square root from a positive trigonometric polynomial.

Lemma 2.1 Let A be a positive trigonometric polynomial invariant under the substitution $\xi \rightarrow-\xi$. A is necessarily of the form

$$
A(\xi)=\sum_{k=0}^{N} a_{k} \cos k \xi, \text { with } a_{k} \in \mathbb{R} .
$$

Then there exists a trigonometric polynomial $B$ of order $N$, i.e.,

$$
B(\xi)=\sum_{k=0}^{N} b_{k} e^{i k \xi}, \text { with } b_{k} \in \mathbb{R}
$$

such that $|B(\xi)|^{2}=A(\xi)$.

According to the above lemma, we can define a trigonometric polynomial $\ell_{N}$ from the square root of $P_{N}(x)$ with $x=(1-\cos \xi) / 2$, that is,

$$
\left|\ell_{N}(\xi)\right|^{2}:=P_{N}\left(\sin ^{2} \frac{\xi}{2}\right), \xi \in[-\pi, \pi] .
$$

Finally, we turn to introduce the symbol of B-spline. This topic has a long story associated with Strang-Fix conditions, approximation of shift invariant subspace, vanishing moments of wavelets, accuracy and construction of Daubechies wavelets. The function $\phi \in L^{2}(\mathbb{R})$ satisfies the Strang-Fix condition of order $N$ if the Fourier transform of $\phi$ admits the equations

$$
\frac{d^{j}}{d \xi^{j}} \hat{\phi}(2 k \pi)=\delta_{k, 0} \delta_{j, 0}
$$

for $0 \leq j \leq N-1$ and $k \in \mathbb{Z}$. Particularly, if $\phi$ is refinable with symbol $m_{\phi}$, then a characterization for the Strang-Fix condition of $\phi$ can be offered in terms of the symbol $m_{\phi}$, that is,

$$
\frac{d^{j}}{d \xi^{j}} m_{\phi}(\pi)=0,0 \leq j \leq N-1 .
$$

In this case, $m_{\phi}$ has the following factorization

$$
m_{\phi}(\xi)=\left(\frac{1+e^{-i \xi}}{2}\right)^{N} \ell_{\phi}(\xi), \xi \in[-\pi, \pi],
$$

where $\ell_{\phi}$ is a $2 \pi$-periodic function. The factor $\left(\frac{1+e^{-i \xi}}{2}\right)^{N}$ is considered as regularity factor since one can measure the index of regularity (smoothness) of $\phi$ according to $N$. Moreover, if we define the function $\beta_{N}$ through

$$
\hat{\beta}_{N}(\xi):=\prod_{k=1}^{\infty}\left(\frac{1+e^{i 2^{-k} \xi}}{2}\right)^{N}, \xi \in \mathbb{R},
$$

one can show that $\beta_{N}$ is just the cardinal B-spline of order $N$, the $N$-fold convolution of the indicator function $\chi_{(0,1)}$ of the interval $(0,1)$, i.e.,

$$
\beta_{N}=\underbrace{\chi_{(0,1)} * \cdots * \chi_{(0,1)}}_{\mathrm{N}} .
$$

It indicates that the factor $\left(\frac{1+e^{-i \xi}}{2}\right)^{N}$ is the symbol of the cardinal B-spline $\beta_{N}$ of order $N$.

\section{Fibonacci-sequence-based Filter Banks}

Note that the low-pass filter $m_{0}$ defined in (4) does not satisfies the necessary condition of orthogonality $\left|m_{0}(\xi)\right|^{2}+$ $\left|m_{0}(\xi+\pi)\right|^{2}=1$, a.e. $\xi \in[-\pi, \pi]$. We define the corresponding dual low-pass filter $\tilde{m}_{0}$ by 


$$
\tilde{m}_{0}(\xi):=\left(\frac{1+e^{-i \xi}}{2}\right)^{N} \frac{\lambda^{2} e^{2 i \xi}+\lambda e^{i \xi}-1}{\lambda^{2}+\lambda-1} \ell_{N}(\xi), \xi \in[-\pi, \pi]
$$

Obviously, $\tilde{m}_{0}$ is also a low-pass filter. Moreover, it can be verified that $\tilde{m}_{0}$ is the dual of $m_{0}$. In fact, direct calculation gives rise to

$$
\begin{aligned}
& m_{0}(\xi) \overline{\tilde{m}_{0}(\xi)}+m_{0}(\xi+\pi) \overline{\tilde{m}_{0}(\xi+\pi)} \\
= & \left|\frac{1+e^{i \xi}}{2}\right|^{2 N}\left|\ell_{N}(\xi)\right|^{2}+\left|\frac{1-e^{i \xi}}{2}\right|^{2 N}\left|\ell_{N}(\xi+\pi)\right|^{2} \\
= & \left(\cos ^{2} \frac{\xi}{2}\right)^{N} P_{N}\left(\sin ^{2} \frac{\xi}{2}\right)+\left(\sin ^{2} \frac{\xi}{2}\right)^{N} P_{N}\left(\cos ^{2} \frac{\xi}{2}\right) \\
= & (1-y)^{N} P_{N}(y)+y^{N} P_{N}(1-y),
\end{aligned}
$$

where $y=\frac{1-\cos \xi}{2}$. Recalling that the Bezout polynomial $P_{N}$ is the solution of the equation (10), it follows that

$$
m_{0}(\xi) \overline{\tilde{m}_{0}(\xi)}+m_{0}(\xi+\pi) \overline{\tilde{m}_{0}(\xi+\pi)}=1 \text {, a.e. } \xi \in[-\pi, \pi] .
$$

As for two high-pass filters $m_{1}$ and $\tilde{m}_{1}$ corresponding to $m_{0}$ and $\tilde{m}_{0}$, respectively, we define $m_{1}$ and $\tilde{m}_{1}$ by

$$
m_{1}(\xi):=e^{i \xi}\left(\frac{1-e^{i \xi}}{2}\right)^{N} \frac{\lambda^{2}+\lambda-1}{\lambda^{2} e^{-2 i \xi}+\lambda e^{-i \xi}-1} \ell_{N}(\xi+\pi), \xi \in[-\pi, \pi]
$$

and

$$
\tilde{m}_{1}(\xi):=e^{i \xi}\left(\frac{1-e^{i \xi}}{2}\right)^{N} \frac{\lambda^{2} e^{2 i \xi}+\lambda e^{i \xi}-1}{\lambda^{2}+\lambda-1} \ell_{N}(\xi+\pi), \xi \in[-\pi, \pi] .
$$

The following theorem claims that $\left\{m_{0}, m_{1}, \tilde{m}_{0}, \tilde{m}_{1}\right\}$ form perfect reconstruction filter banks. Theorem 3.1 Supposed that the $2 \pi$-periodic functions $m_{0}, m_{1}, \tilde{m}_{0}, \tilde{m}_{1}$ are defined by (4), (13), (12), (14). Then $\left\{m_{0}, m_{1}, \tilde{m}_{0}, \tilde{m}_{1}\right\}$ are perfect reconstruction filter banks.

proof: To verify (5), it suffices to check the following equations

$$
\begin{aligned}
& m_{0}(\xi) \overline{\tilde{m}_{1}(\xi)}+m_{0}(\xi+\pi) \overline{\tilde{m}_{1}(\xi+\pi)}=0, \text { a.e. } \xi \in[-\pi, \pi], \\
& m_{1}(\xi) \overline{\tilde{m}_{0}(\xi)}+m_{1}(\xi+\pi) \overline{\tilde{m}_{0}(\xi+\pi)}=0, \text { a.e. } \xi \in[-\pi, \pi],
\end{aligned}
$$

and

$$
m_{1}(\xi) \overline{\tilde{m}_{1}(\xi)}+m_{1}(\xi+\pi) \overline{\tilde{m}_{1}(\xi+\pi)}=1 \text {, a.e. } \xi \in[-\pi, \pi] .
$$

For the convenience of readers, the calculation details are as follows. To obtain the equation (15), recalling the definition of $m_{0}$ and $\tilde{m}_{0}$, we get

$$
\begin{aligned}
& m_{0}(\xi) \overline{\tilde{m}_{1}(\xi)}+m_{0}(\xi+\pi) \overline{\tilde{m}_{1}(\xi+\pi)} \\
= & \left(\frac{1+e^{-i \xi}}{2}\right)^{N} \frac{\lambda^{2}+\lambda-1}{\lambda^{2} e^{-2 i \xi}+\lambda e^{-i \xi}-1} e^{-i \xi}\left(\frac{1-e^{-i \xi}}{2}\right)^{N} \frac{\lambda^{2} e^{-2 i \xi}+\lambda e^{-i \xi}-1}{\lambda^{2}+\lambda-1} \ell_{N}(\xi) \overline{\ell_{N}(\xi+\pi)} \\
& +\left(\frac{1-e^{-i \xi}}{2}\right)^{N} \frac{\lambda^{2}+\lambda-1}{\lambda^{2} e^{-i 2 \xi}-\lambda e^{-i \xi}-1}\left(-e^{-i \xi}\right)\left(\frac{1+e^{-i \xi}}{2}\right)^{N} \frac{\lambda^{2} e^{-2 i \xi}-\lambda e^{-i \xi}-1}{\lambda^{2}+\lambda-1} \ell_{N}(\xi+\pi) \overline{\ell_{N}(\xi+2 \pi)} \\
= & 0 .
\end{aligned}
$$


Similarly, the equation (16) can be obtained from the following calculation

$$
\begin{aligned}
& m_{1}(\xi) \overline{\tilde{m}_{0}(\xi)}+m_{1}(\xi+\pi) \overline{\tilde{m}_{0}(\xi+\pi)} \\
= & e^{i \xi}\left(\frac{1-e^{i \xi}}{2}\right)^{N} \frac{\lambda^{2}+\lambda-1}{\lambda^{2} e^{-2 i \xi}+\lambda e^{-i \xi}-1} \ell_{N}(\xi+\pi) \frac{\lambda^{2} e^{-2 i \xi}+\lambda e^{-i \xi}-1}{\lambda^{2}+\lambda-1}\left(\frac{1+e^{i \xi}}{2}\right)^{N} \overline{\ell_{N}(\xi)} \\
& -e^{i \xi}\left(\frac{1+e^{i \xi}}{2}\right)^{N} \frac{\lambda^{2}+\lambda-1}{\lambda^{2} e^{-2 i \xi}-\lambda e^{-i \xi}-1} \ell_{N}(\xi+2 \pi) \frac{\lambda^{2} e^{-2 i \xi}-\lambda e^{-i \xi}-1}{\lambda^{2}+\lambda-1}\left(\frac{1-e^{i \xi}}{2}\right)^{N} \overline{\ell_{N}(\xi+\pi)} \\
= & e^{i \xi}\left(\frac{1-e^{-2 i \xi}}{4}\right)^{N} \ell_{N}(\xi+\pi) \overline{\ell_{N}(\xi)}-e^{i \xi}\left(\frac{1-e^{-2 i \xi}}{4}\right)^{N} \ell_{N}(\xi+2 \pi) \overline{\ell_{N}(\xi+\pi)} \\
= & 0 .
\end{aligned}
$$

To obtain the equation (17), we get

$$
\begin{aligned}
& m_{1}(\xi) \overline{\tilde{m}_{1}(\xi)}+m_{1}(\xi+\pi) \overline{\tilde{m}_{1}(\xi+\pi)} \\
= & e^{i \xi}\left(\frac{1-e^{i \xi}}{2}\right)^{N} \frac{\lambda^{2}+\lambda-1}{\lambda^{2} e^{-2 i \xi}+\lambda e^{-i \xi}-1} \ell_{N}(\xi+\pi) e^{-i \xi}\left(\frac{1-e^{-i \xi}}{2}\right)^{N} \frac{\lambda^{2} e^{-2 i \xi}+\lambda e^{-i \xi}-1}{\lambda^{2}+\lambda-1} \overline{\ell_{N}(\xi+\pi)} \\
& -e^{i \xi}\left(\frac{1+e^{i \xi}}{2}\right)^{N} \frac{\lambda^{2}+\lambda-1}{\lambda^{2} e^{-2 i \xi}-\lambda e^{-i \xi}-1} \ell_{N}(\xi+2 \pi)\left(-e^{i \xi}\right)\left(\frac{1+e^{-i \xi}}{2}\right)^{N} \frac{\lambda^{2} e^{-2 i \xi}-\lambda e^{-i \xi}-1}{\lambda^{2}+\lambda-1} \overline{\ell_{N}(\xi+2 \pi)} \\
= & \left(\frac{1-e^{-i \xi}}{2}\right)^{N} P_{N}\left(\cos ^{2} \frac{\xi}{2}\right)\left(\frac{1-e^{i \xi}}{2}\right)^{N}+\left(\frac{1+e^{-i \xi}}{2}\right)^{N} P_{N}\left(\sin ^{2} \frac{\xi}{2}\right)\left(\frac{1+e^{i \xi}}{2}\right)^{N} .
\end{aligned}
$$

Using the variable change $x=(1-\cos \xi) / 2$, it follows

$$
\begin{aligned}
& m_{1}(\xi) \overline{\tilde{m}_{1}(\xi)}+m_{1}(\xi+\pi) \overline{\tilde{m}_{1}(\xi+\pi)} \\
= & \left|\frac{1-e^{i \xi}}{2}\right|^{2 N} \ell_{N}(\xi+\pi) \overline{\ell_{N}(\xi+\pi)}+\left|\frac{1+e^{i \xi}}{2}\right|^{2 N} \ell_{N}(\xi+2 \pi) \overline{\ell_{N}(\xi+2 \pi)} \\
= & \left|\frac{1-e^{i \xi}}{2}\right|^{2 N} P_{N}\left(\cos ^{2} \frac{\xi}{2}\right)+\left|\frac{1+e^{i \xi}}{2}\right|^{2 N} P_{N}\left(\sin ^{2} \frac{\xi}{2}\right) \\
= & \left|\frac{1-e^{i \xi}}{2}\right|^{2 N} P_{N}(1-x)+\left|\frac{1+e^{i \xi}}{2}\right|^{2 N} P_{N}(x) \\
= & x^{N} P_{N}(1-x)+(1-x)^{N} P_{N}(x)=1 .
\end{aligned}
$$

This proof of this theorem is completed.

\section{Discussion and Conclusion}

The properties of the scaling function $\phi$ and its dual scaling function $\tilde{\phi}$ are completely determined by the filters $m_{0}$ and $\tilde{m}_{0}$ since they are defined respectively in frequency domain by an infinite product (7). We need to explain the well defined function $\hat{\phi}$ and $\hat{\tilde{\phi}}$, which is equivalent to check the convergence

$$
\begin{aligned}
|m(\xi)| & \leq 1+|m(\xi)-1| \leq 1+\left|\sum_{n} h_{n}\left(1-e^{i n \xi}\right)\right| \\
& \leq 1+\sum_{n}\left|h_{n}\right| 2\left|\sin \frac{n \xi}{2}\right| \leq 1+|\xi| \sum_{n}\left|h_{n}\right||n| \\
& \leq e^{c|\xi|}
\end{aligned}
$$

with $c=\sum_{n}\left|h_{n}\right||n|$. Then

$$
\prod_{k=1}^{\infty}\left|m\left(2^{-k} \xi\right)\right| \leq \exp \left(\sum_{k=1}^{\infty} c 2^{-k}|\xi|\right)=e^{c|\xi|}
$$


Therefore, the infinite products $\prod_{k=1}^{\infty} m_{0}\left(2^{-k} \xi\right)$ and $\prod_{k=1}^{\infty} \tilde{m}_{0}\left(2^{-k} \xi\right)$ converge absolutely and uniformly on compact sets of $\mathbb{R}$. The functions $\hat{\phi}$ and $\hat{\tilde{\phi}}$ are in $C(\mathbb{R})$.

In this note, we adopt the Hadamard product of the Fibonacci sequence and a geometric sequence to construct a type of Fibonacci-based bi-orthogonal filter banks. These filters are essentially rational functions, which have potential applications in system identification in frequency domain and signal processing.

\section{Acknowledgements}

The authors would like to thank the Referees and Editors for carefully reading the manuscript and making several useful suggestions. This work was supported in part by NSFC No.11571083 and No.11971178 and Key $\mathrm{R}$ and D Program of Guangdong science and technology plan (No.2019B020223001 and No.2019B020214001-1) ;Gouangdong Provincial Special Fund For Modern Agriculture Industry Technology Innovation Teams (No.2019LM119).

\section{References}

[1] A. Cohen, I. Daubechies, Non-separable bidimensional wavelet bases, Rev. Mat. Iberoamericana 9 (1993), 51137.

[2] A. Croisier, D. Esteban and C. Galand, Perfect channel splitting by use of interpolation/ decimation/tree decomposition techniques, in Int. Conf. on Info. Sciences and Systems, 443-446, Patras, Greece, August 1976.

[3] I. Daubechies, Ten Lectures on Wavelets, CBMS, 61, SIAM, Philadelphia, 1992.

[4] R. E. Edwards, Fourier Series, A Mordern Introduction, Volumn 2, GTM 64, Springer-Verlag New York,Inc., 1979.
[5] C. Heil and D. Colella, Matrix refinement equation: existence and uniqueness, J. Fourier Anal. Appl. 2 (1996), 363-377.

[6] J. Kovacevic and M. Vetterli, Nonseparable multidimensional perfect reconstruction filter banks and wavelet bases for $R^{n}$, IEEE Tran. on Information Theory 38(1992), 533-555.

[7] S. Mallat, A Wavelet Tour of Signal Processing, Academic Press, 3rd edition, 2008.

[8] T. Q. Nguyen and P. P. Vaidyanathan, Two-channel perfect reconstruction FIR QMF structure which yield linear phase FIR analysis and synthesis filters, IEEE Trans. Acoustics, Speech and Signal Proc. 37 (1989), 676-690.

[9] M. J. Smith and T. P. Barnwell, A procedure for designing exact reconstruction filter banks for tree structured subband coders, in Proc. IEEE Int. Conf. Acoust. Speech and Signal Proc., San Diego, CA, March 1984.

[10] P. P. Vaidyanathan, Multirate Systems and Filter Banks, Prentice Hall, Englewood Cliffs, New Jersey, 1993.

[11] P. Przemyslaw, H. James, A. Lindenmayer, Systems, Fractals, and Plants, Lecture Notes in Biomathematics, Springer-Verlag, ISBN 0387970924,1992.

[12] Ahamad, Mohd Vasim, et al. An Improved Playfair Encryption Technique Using Fibonacci Series Generated Secret Key. International Journal of Engineering and Technology 7.4 .5 (2018): 347-351.

[13] Zhang, Yingge, et al. Multiplanar Fourier-transform phase-shifting digital holography with a generalized Fibonacci lens. Laser Physics Letters, 2019, 16.5: 055201.

[14] Ghosh, Nabakumar. Fibonacci numbers in real life applications. Mugberia Gangadhar Mahavidyalaya, 1 (2018): 62-69.

[15] Singh, P., et al. Fractal and periodical biological antennas: Hidden topologies in DNA, wasps and retina in the eye. Soft Computing Applications. Springer, Singapore, 2018. 113-130. 